\title{
REVIEW
}

\section{Significance, definition, classification and risk factors of chronic kidney disease in South Africa}

\author{
A M Meyers, MB BCh, FCP (SA), Cert Nephrology (SA), FRCP (Lond) \\ Donald Gordon Medical Centre, Klerksdorp Hospital, and National Kidney Foundation of South Africa, Johannesburg, South Africa
}

Corresponding author: A M Meyers (nkfsa@mweb.co.za)

Renal dysfunction or chronic kidney disease (CKD) is found in $10 \%$ of the global population and is classified into five stages according to the estimated glomerular filtration rate (eGFR). No matter where a patient lives, estimation of the GFR is mandatory for decision-making and obtained by the simple measurement of a serum creatinine level. The objective of diagnosing CKD lies in its future prevention, early detection and proper treatment, which will prevent or delay functional deterioration.

Primary hypertension (PH) occurs in $25 \%$ of South Africa (SA)'s black population and is the putative cause of stage 5 CKD in $40-60 \%$ of these patients. Moreover, in this group, stage 5 CKD occurs at a relatively young age (35 - 45 years) compared with other population groups in whom stage 5 CKD resulting from PH usually occurs between 60 and 70 years of age. In the cohort study, PH has been found in 12 - $16 \%$ of black school learners (mean age 17 years) compared with 1.8 - 2\% of other ethnic groups (mixed race, Asian, white). End-stage renal failure (ESRF) is the fifth most common cause of death in SA, excluding post-traumatic cases. In addition, undiagnosed or poorly controlled PH is a potent risk factor for other cardiovascular disease (CVD), e.g. congestive cardiac failure, myocardial infarction, stroke. Significant protein is also associated with CVD and protein $>1 \mathrm{~g} / \mathrm{d}$ is a significant risk factor for ESRF.

S Afr Med J 2015;105(3):233-236. DOI:10.7196/SAMJ.9412

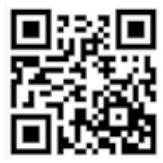

\section{Chronic kidney disease (CKD) matters}

- $\mathrm{CKD}$ is an important disease group that threatens health.

- End-stage kidney disease (ESKD) has led to an increasing number of dialysis and transplanted patients worldwide, posing a massive burden on health economics, especially in developing countries.

- ESKD resulting from hypertensive and/ or diabetic nephropathy is increasing globally, especially in the elderly. However, hypertension with renal dysfunction is an even greater problem in South Africa (SA), occurring in relatively young black patients who often also have severe target organ damage (heart and kidney).

- The development of CKD is associated with atherosclerosis caused by lifestylerelated diseases such as obesity, hypertension and diabetes. Even in the absence of lifestyle diseases, CKD is a risk factor for cardiovascular disease (CVD).

- $\mathrm{CKD}$ is an important disease group that is most likely to cause CVD, hospitalisation or death, thus threatening the health of South Africans.

- In an article by Mayosi et al., ${ }^{[1]}$ end-stage renal failure (ESRF) has been shown to be the fifth highest cause of non-traumatic death in SA.

Only estimated or deduced statistics exist for SA. However, with our burden of hypertensive ESKD, HIV nephropathy and other poverty-related disorders, it is highly likely that the sub-Saharan burden

of CKD is far higher than that in developed countries. In Japan, with a population of $>104000000$, the prevalence of CKD was

Table 1. Prevalence of adult CKD in a developed country (Japan). The number of patients with stage 1 or 2 CKD was estimated according to the presence of pathological proteinuria. Patients on dialysis $(N=275000)$ were excluded

\begin{tabular}{llll}
\hline CKD stage & eGFR & Patients, $\boldsymbol{n}^{*}$ & CKD, \% (approximately) \\
\hline 1 & $\geq 90$ & 605313 & 5 \\
2 & $60-89$ & 1708876 & 13 \\
3 & $30-59$ & 10743236 & 80 \\
4 & $15-29$ & 191045 & 1.9 \\
5 & $<15$ & 45524 & 0.1 \\
$\begin{array}{l}\text { eGFR = estimated glomerular filtration rate. } \\
\text { Total = 13 293 985 patients. }\end{array}$ & &
\end{tabular}

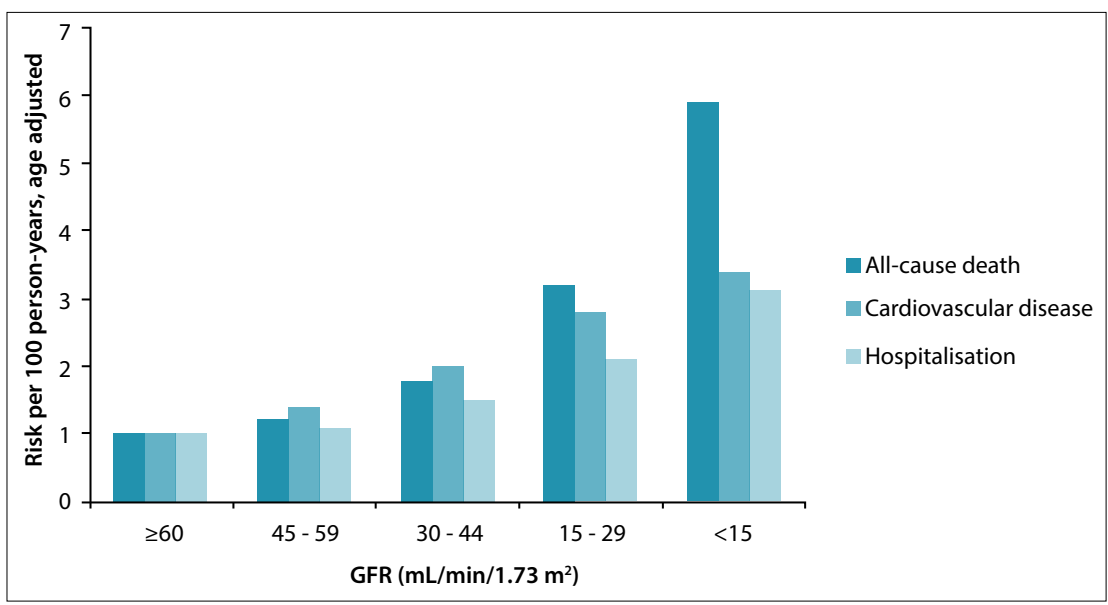

Fig. 1. The poorer the kidney function, the higher the risk of all-cause death, cardiovascular disease and hospitalisation. (GFR = glomerular filtration rate.) 
13.3 million (i.e. $12.9 \%$ of the population) (Table 1). ${ }^{[2]}$

A decline in kidney function is an important risk factor for CVD. The poorer the kidney function, the higher the risk of CVD. Besides CVD, a large-scale epidemiological investigation revealed that relative risks for total mortality or hospitalisation rise irrespective of cause in proportion to the degree of reduction in kidney function (Fig. 1). ${ }^{[3]}$

Fig. 2 shows the relative risk of death from cardiovascular events according to the presence or absence of proteinuria and kidney function level. The relative risk was regarded as 1.0 for the cohort in the general health examination. This group comprised 30704

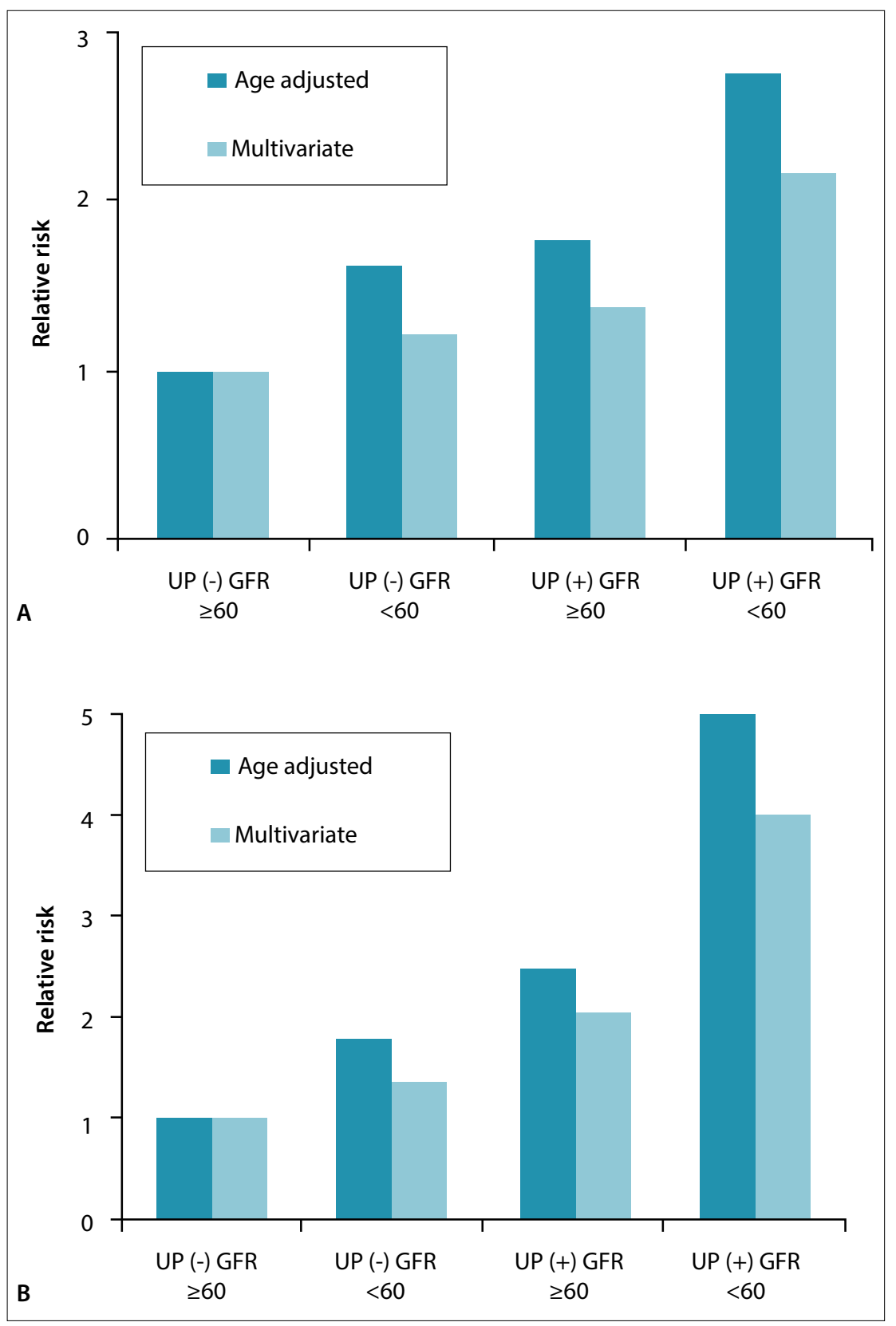

Fig. 2. (A): Men. (B): Women. The relative risk of cardiovascular death associated with proteinuria and CKD. (General population: men 30764 , women 60668 , age 40 - 79 years; reference: GFR $\geq 60$, proteinuria ( - ).) Data, with modification, from Irie et al. ${ }^{[4]}$ (UP = proteinuria; GFR = glomerular filtration rate.) men and 60668 women aged 40 - 79 years with an eGFR $\geq 60 \mathrm{~mL} / \mathrm{min} / 1.73 \mathrm{~m}^{2}$ and no proteinuria. The age-adjusted relative risk of CVD mortality is shown for men and women.

\section{Definition and \\ classification of CKD}

- $\mathrm{CKD}$ is diagnosed by proteinuria, albuminuria, haematuria (not all cases are renal) or decreased glomerular filtration rate (GFR).

- CKD stages are classified according to the GFR. Most laboratories give a calculated GFR, which is derived from the serum creatinine value. The most commonly given value is the estimated GFR (eGFR).

- CKD should be appropriately treated, depending on its stage (Fig. 3).

Kidney Disease Improving Global Outcomes divides stage 3 into $3(\mathrm{a})$ and $3(\mathrm{~b})$ to facilitate therapeutic intervention in 3(b) patients in whom kidney dysfunction is easily aggravated. Furthermore, albuminuria is categorised as normal $(<30 \mu \mathrm{mol} / \mathrm{d})$, or positive $(30-299 \mu \mathrm{mol} / \mathrm{d})$, and proteinuria as normal $(<150 \mu \mathrm{mol} / \mathrm{d})$, mild $(150-490 \mu \mathrm{mol} / \mathrm{d})$ and severe $(\geq 300 \mu \mathrm{mol} / \mathrm{d})$, i.e. categories A1, A2 and A3.

\section{Diagnostic criteria of CIND}

CKD is defined and described in Table 2 and Fig. 3. It includes all morbid conditions associated with reduced kidney function indicated by the GFR or persistent findings that suggest kidney damage.

Instances of kidney damage:

- urinary abnormalities such as proteinuria, including microalbuminuria

- abnormalities of imaging tests, such as single kidney or polycystic kidney

- abnormalities of blood biochemistry, such as those indicating kidney dysfunction, including abnormal glomerular and tubular dysfunction

- abnormalities of histological findings.

The eGFR as the measurement of creatinine levels in blood provides vital information on the management of CKD. eGFR should, however, not be accepted as the single only measurement of kidney function (Table 3).

\section{CKD: High-risk groups}

- The most important risk factor in SA's black population for CKD is the

\section{Table 2. CKD definition}

A. Obvious kidney damage shown by urinalysis, blood chemistry, images or pathology of the kidney. The presence of albuminuria or proteinuria is especially important. Note: Kidney cysts in the elderly do not represent CKD unless they are the result of autosomal-dominant polycystic kidney disease B. GFR as a risk factor for ESRF: $\leq 60 \mathrm{~mL} / \mathrm{min} / 1.73 \mathrm{~m}^{2}$ for ages 40 - 49 years and $\leq 40$ for ages $\geq 70$ years Persistent evidence of A and/or B for $\geq 3$ months 
development of essential hypertension. In SA, hypertension develops much earlier than in developed areas of the world, is more severe, usually not diagnosed early, and often poorly treated. All of this is compounded by poor patient compliance.

- There are also the usual risk factors for the development of CKD: ageing, family history of CKD (including essential hypertension and type 2 diabetes mellitus (DM)), habitual use of non-steroidal antiinflammatory drugs (NSAIDs), history of abnormal urinary findings and/or abnormal kidney function, abnormal morphology of the kidney, dyslipidaemia, hyperuricaemia, obesity, metabolic syndrome, auto-immune disorders, kidney infections, nephrotic syndrome and kidney stones.
- In the SA context, the HIV epidemic is another relevant factor in the development of CKD ( $10 \%$ of these patients may develop advanced $\mathrm{CKD}$ ).

- Pregnancy-related factors are also important. Fetuses whose mothers abuse alcohol and/ or cigarettes during pregnancy or who are malnourished or immunocompromised are at risk. Infants born to these mothers have a high incidence of low birthweight $(\leq 2 \mathrm{~kg})$ and a high occurrence of subsequent severe CKD.

- As a safeguard against the development of $\mathrm{CKD}$, the following basic strategies must be implemented:

- All individuals with a positive family history (i.e. parental or sibling) of hypertension or any other familial renal disease should have their blood

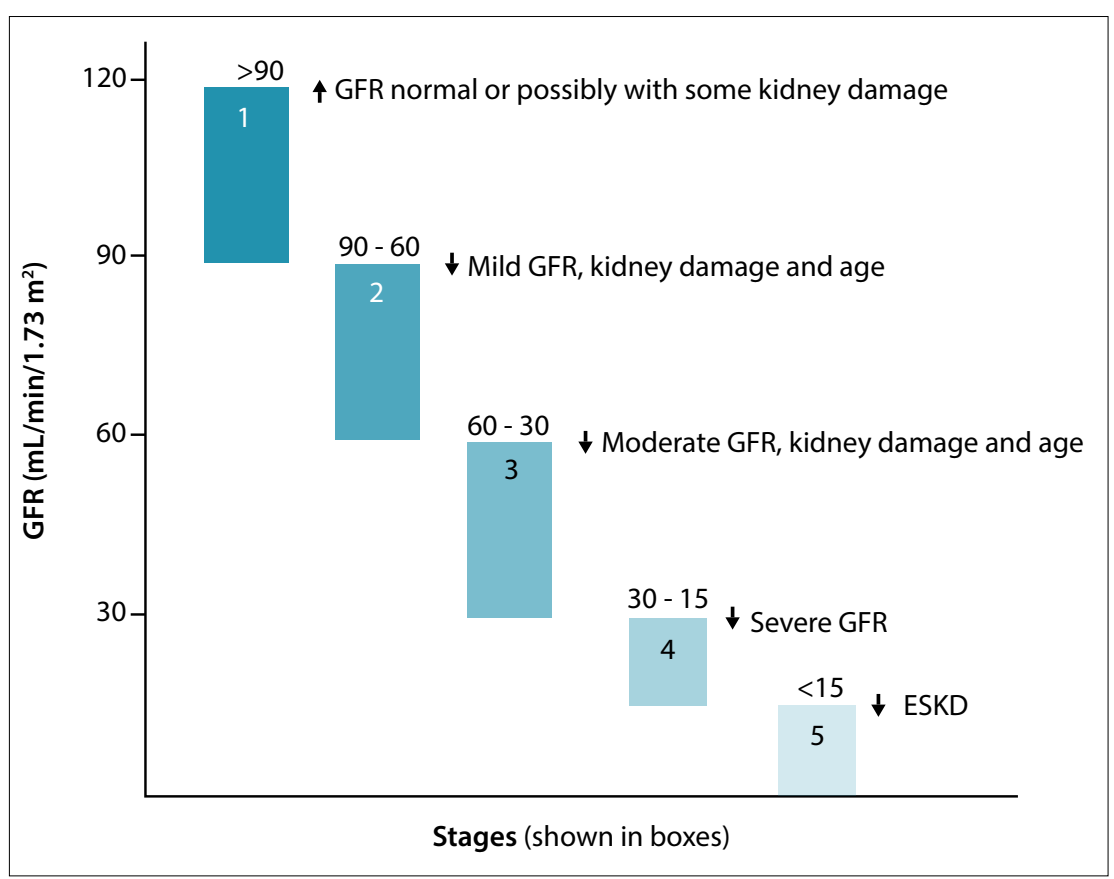

Fig. 3. Classification of the stages of CKD (generally referred to as CKD \# where \# is classified according to the eGFR). $(G F R=$ glomerular filtration rate; ESKD = end-stage kidney disease. $)$ pressure (BP) checked annually from an early age (e.g. 20 years onwards) and managed appropriately. Checking of $\mathrm{BP}$ should always be accompanied by urine dipstick testing, and abnormalities must be assessed by a doctor.

- The same applies to DM patients, but checking should start at an older age (e.g. 35 years).

- Major risk factors for the abovementioned two disorders are lifestyle eating aberrations and, in particular, the excessive intake of dietary sodium. Obesity, which is often seen in SA as a societal attraction, must be condemned and prevented.

- The HIV epidemic must be controlled by all means possible.

- One of the most important causal factors of kidney function deterioration in healthy people is ageing. From about the age of 50 - 55 years, the GFR reduces by approximately $1.2-1.5 \mathrm{~mL} / \mathrm{min} /$ year. This calls for careful drug administration with advancing age, especially drugs that are largely excreted by the kidneys.

- If patients with proteinuria smoke, are obese, or develop hypertension or an impaired glucose tolerance, CKD advances at a more rapid rate. Men are at particular risk; they should be put on stricter treatment regimens and be required to modify their lifestyle.

- Obesity (especially morbid obesity) is a risk factor for proteinuria, which can result in ESKD - even in the absence of diabetes or hypertension (especially in men).

- Dyslipidaemia is a risk factor for CVD. Although based on scant evidence, it has been suggested that dyslipidaemia may promote ESKD. Conversely, an increase in proteinuria is associated with an increased incidence and severity of dyslipidaemia, probably resulting in further proteinuria and progressive CKD.

- Hyperuricaemic patients frequently suffer from kidney disorders and vice versa, and

\section{Table 3. Interpretation of serum creatinine}

- Creatinine - not urea - is of pivotal importance in assessing GFR

- If eGFR testing reveals stage $2 \mathrm{CKD}$, repeat test twice per year. If stage 3 or 4 , repeat test three or four times per year

- Factors other than GFR can influence levels of serum creatinine:

- age (progressive decrease in GFR is physiological)

- muscle bulk (lower in females, increased in males)

- diet, e.g. vegetarian

- some drugs, e.g. cimetidine, result in a falsely high creatinine

- Examples of major pitfalls:

- a muscular man with a serum creatinine of $120 \mu \mathrm{mol} / \mathrm{L}$ may have a normal GFR

- conversely, a thin elderly woman with a serum creatinine of $90 \mu \mathrm{mol} / \mathrm{L}$ may have reduced eGFR, e.g. $<40 \mathrm{~mL} / \mathrm{min} / 1.73 \mathrm{~m}{ }^{2}$

- Important practice point:

- A serum creatinine of $\geq 150 \mu \mathrm{mol} / \mathrm{L}$ in females or $160 \mu \mathrm{mol} / \mathrm{L}$ in males indicates serious underlying renal dysfunction. These patients must be referred to a nephrologist 
stages 4 - 5 CKD patients tend to have hyperuricaemia. There is accumulating evidence, however, that hyperuricaemia is an independent risk factor for atherosclerosis and progressive CKD.

- To summarise, all individuals with a positive family history of renal disorders are potentially at high risk and should be regarded as such.

\section{Sixteen dos and don'ts in the basic management of CKD patients}

1. CKD is defined as either:

- a kidney disorder (e.g. proteinuria) with or without impairment of GFR, and/or

- decreased GFR, i.e. $\leq 60 \mathrm{~mL} / \mathrm{min} / 1.73 \mathrm{~m}^{2}$ lasting for $>3$ months.

2. eGFR is calculated using the so-called modification of diet in renal disease (MDRD) formula:

eGFR $\mathrm{mL} / \mathrm{min} / 1.73 \mathrm{~m}^{2}=32788 \downarrow \times$ serum creatinine $\mathrm{e}^{-1.154} \times$ age $^{-0.203}$ $(\times 1.210$ if black and $\times 0.742$ if female)

3. CKD is a critical risk factor for the development of CVD and ESKD.

4. A CKD patient should be managed by a multidisciplinary team of primary care physicians and nephrologists.

5. It is desirable that patients with the following are referred to nephrologists:

- proteinuria $0.5 \mathrm{~g} / \mathrm{g}$ creatinine or higher; or on dipstick $\geq 2+$

- eGFR of $<50 \mathrm{~mL} / \mathrm{min} / 1.73 \mathrm{~m}^{2}$

- proteinuria plus haematuria of $\geq 1+$.

6. The treatment goal in cases of proteinuria is to achieve levels of $<0.5 \mathrm{~g} / \mathrm{g}$ creatinine, i.e. $<500 \mu \mathrm{g} / 24 \mathrm{~h}$.

7. CKD management should be started with modification of lifestyle, i.e. smoking cessation, salt restriction and improvement of obesity.

8. The goal of BP control is $<130 / 80 \mathrm{mmHg}$ and should be achieved gradually. Automatic home BP devices should be used whenever possible, and BP charts maintained and checked by doctors.

9. Antihypertensive agents of first choice are angiotensin-converting enzyme (ACE) inhibitors or angiotensin receptor blockers (ARBs).
Combination with other antihypertensives should be as required but ACE I or A II RB should always be combined with a thiazide diuretic in black patients. Thiazide diuretics (other than furosemide) are inactive if the GFR is $<30 \mathrm{~mL} / \mathrm{min} / 1.73 \mathrm{~m}^{2}$.

10. When ACE I or ARBs are used, a physician must be aware of the possible risk of a slight rise in the creatinine level and the possible occurrence of hyperkalaemia with stage 3(b), 4 or 5 CKD patients.

11. In diabetic nephrology, the target level of $\mathrm{HbA}_{1 \mathrm{C}}$ should be approximately $6.5 \%$ to control the blood glucose level

12. The low-density lipoprotein cholesterol should be controlled at $\leq 2.5 \mathrm{mmol} / \mathrm{L}$

13. The primary physician should consult a nephrologist when renal anaemia is suspected.

14. Physicians should consult a nephrologist whenever erythropoiesisstimulating agents are considered.

15. Physicians should reduce the dose or extend the administration interval, depending on kidney function, when administering drugs that are renally excreted.

16. NSAIDs, contrast media and diuretics are high-grade risk factors for a decline (often permanent) in kidney function in patients with advanced CKD. The same occasionally applies with the use of ACE I and A II RB in stages 4 and 5 CKD, especially in elderly, hypertensive or diabetic populations.

References

1. Mayosi BM, Flisher AJ, Lalloo UG, Sitas F, Tollman SM, Bradshaw D. The burden of noncommunicable diseases in South Africa. Lancet 2009;374(9693):934-947. [http://dx.doi.org/10.1016/ S0140-6736(09)61087-4]

2. Japanese Society of Nephrology. Japanese Guidelines. Clin Exp Nephrol 2009;13(3):192-248. [http:// dx.doi.org/10.1007/s10157-009-0188-0] http://link.springer.com/article/10.1007/s10157-009-0131-4/ fulltext.html (accessed 30 January 2015).

3. Go AS, Chertow GM, Fan D, McCulloch CE, Hsu CY. Chronic kidney disease and the risks of death, cardiovascular events, and hospitalization. N Engl I Med 2004:351:1296-1305.

4. Irie F, Iso H, Sairenchi $\mathrm{T}$, et al. The relationships of proteinuria, serum creatinine, glomerular filtration rate with cardiovascular disease mortality in Japanese general population. Kidney Int 2006;69(7):1264-
ration rate wi 